\title{
Pavelka-style fuzzy logic for attribute implications
}

\author{
Radim Bělohlávek Vilém Vychodil \\ Dept. Computer Science, Palacký University, Tomkova 40, CZ-779 00, Olomouc, Czech Republic \\ Email: \{radim.belohlavek, vilem.vychodil\}@upol.cz
}

\begin{abstract}
We present Pavelka-style fuzzy logic for reasoning about attribute implications, i.e. formulas $A \Rightarrow B$ known also as association rules and functional dependencies. Fuzzy attribute implications allow for two different interpretations, namely, in data tables with graded (fuzzy) attributes and in data tables over domains with similarity relations. In the first interpretation, $A \Rightarrow B$ reads "each object having all attributes from $A$ has also all attributes from $B$ ". In the second interpretation, $A \Rightarrow B$ reads "any two table rows which have similar values on attributes from $A$ have similar values on attributes from $B$ ". The axioms of our logic are inspired by well-known Armstrong axioms but the logic allows us to infer partially true formulas from partially true formulas. We prove soundness and completeness of our logic in graded style, i.e. we prove that a degree to which an attribute implication $A \Rightarrow B$ semantically follows from a collection $T$ of partially true attribute implications equals a degree to which $A \Rightarrow B$ is provable from $T$.
\end{abstract}

Keywords: attribute dependency, fuzzy logic, attribute implication, Armstrong axioms, graded completeness

\section{Introduction}

Reasoning about rules $A \Rightarrow B$ where $A$ and $B$ are collections of attributes plays a crucial role in several areas of computer science and data engineering in particular. Two most important areas are databases where rules $A \Rightarrow B$ are called functional dependencies [12], and data mining where the rules are called association rules [15].

In our previous papers $[2,3,4,5,6]$, we studied the rules $A \Rightarrow B$ from the point of view of fuzzy approach. We presented two kinds of semantics for the rules. First, a semantics in tables where a table entry contains a truth degree to which an object (row) has an attribute (column), see $[2,4]$. Second, a semantics in tables which extend ordinary database tables in that each domain is equipped with a similarity relation $[6,7]$. We proved that both of the semantics have the same notion of semantic entailment. We developed several issues, e.g. description of non-redundant bases and an algorithm for computing the bases, see $[2,7]$. Furthermore, we developed a logical calculus in an ordinary style, i.e. provability is defined from an ordinary set of attribute implications (which are, therefore assumed to be fully valid) using ordinary deduction rules, see e.g. [3, 5].

Such a notion, however, does not directly capture the fact that an attribute implication may be only partially true, i.e. we may assume its validity only to some degree, say 0.7 , not only 0 or 1 . In addition to that, an attribute implication can follow semantically from other attribute implications (valid partially or completely) in a degree, say 0.9 , not only 0 or 1 . In this paper, we develop a logic which captures both inference from partially true attribute implications and semantic entailment in degrees. Our logic fits the framework of graded style (Pavelkastyle) fuzzy logic, see e.g. [9, 10, 14, 13]. The axioms of our logic are inspired by well-known Armstrong axioms from databases. We prove soundness and completeness of our logic.

\section{Preliminaries}

Due to lack of space, we just survey basic notions and refer to, e.g., $[1,9,10]$ for further details and properties. Our basic structure of truth degrees is a complete residuated lattice with a hedge, i.e., an algebra $\mathbf{L}=\langle L, \wedge, \vee, \otimes, \rightarrow$ $\left.,{ }^{*}, 0,1\right\rangle$ such that $\langle L, \wedge, \vee, 0,1\rangle$ is a complete lattice with 0 and 1 being the least and greatest element of $L$, respectively; $\langle L, \otimes, 1\rangle$ is a commutative monoid (i.e. $\otimes$ is commutative, associative, and $a \otimes 1=1 \otimes a=a$ for each $a \in L) ; \otimes$ and $\rightarrow$ satisfy so-called adjointness property: $a \otimes b \leq c$ iff $a \leq b \rightarrow c$; for each $a, b, c \in L$; hedge * satisfies (i) $1^{*}=1$, (ii) $a^{*} \leq a$, (iii) $(a \rightarrow b)^{*} \leq a^{*} \rightarrow b^{*}$, (iv) $a^{* *}=a^{*}$, for all $a, b \in L$. Elements $a$ of $L$ are called truth degrees. $\otimes$ and $\rightarrow$ are (truth functions of) "fuzzy conjunction" and "fuzzy implication". Hedge * is a (truth function of) logical connective "very true", see $[10,11]$. Properties (i)-(iv) have natural interpretations, e.g. (iii) can be read: "if $a \rightarrow b$ is very true and if $a$ is very true, then $b$ is very true", etc.

A common choice of $\mathbf{L}$ is a structure with $L=[0,1]$ (unit interval), $\wedge$ and $\vee$ being minimum and maximum, $\otimes$ being a left-continuous t-norm with the corresponding $\rightarrow$. Three most important pairs of adjoint operations on the unit interval are: Łukasiewicz $(a \otimes b=\max (a+b-1,0)$, $a \rightarrow b=\min (1-a+b, 1))$, Gödel: $(a \otimes b=\min (a, b)$, $a \rightarrow b=1$ if $a \leq b, a \rightarrow b=b$ else), Goguen (product): $\left(a \otimes b=a \cdot b, a \rightarrow b=1\right.$ if $a \leq b, a \rightarrow b=\frac{b}{a}$ else). Ex- 
amples of finite structures: Take a finite subset $L \subseteq[0,1]$ which is closed under Łukasiewicz or Gödel operations. If we take $L=\{0,1\}$, this gives us a two-element Boolean algebra (structure of truth degrees of classical logic). Two boundary cases of hedges are (i) identity, i.e. $a^{*}=a$ ( $a \in L)$; (ii) globalization: $a^{*}=1$ if $a=1, a^{*}=0$ else.

Given $\mathbf{L}$ which serves as a structure of truth degrees, we define usual notions: an L-set (fuzzy set) $A$ in universe $U$ is a mapping $A: U \rightarrow L, A(u)$ being interpreted as "the degree to which $u$ belongs to $A$ "; $A$ is also denoted by $A=\{a / u, \ldots\}$ where $a=A(u)$. Let $\mathbf{L}^{U}$ denote the collection of all $\mathbf{L}$-sets in $U$. The operations with L-sets are defined componentwise. For instance, intersection of $\mathbf{L}$-sets $A, B \in \mathbf{L}^{U}$ is an $\mathbf{L}$-set $A \cap B$ in $U$ such that $(A \cap B)(u)=A(u) \wedge B(u)$ for each $u \in U$, etc. For $a \in L$ and $A \in \mathbf{L}^{U}$, we define $\mathbf{L}$-sets $a \otimes A$ (a-multiple of $A$ ) and $a \rightarrow A$ ( $a$-shift of $A)$ by $(a \otimes A)(u)=a \otimes A(u)$, $(a \rightarrow A)(u)=a \rightarrow A(u)(u \in U)$. Given $A, B \in \mathbf{L}^{U}$, we define a subsethood degree $S(A, B)=\bigwedge_{u \in U}(A(u) \rightarrow B(u))$, which generalizes the classical subsethood relation $\subseteq$. Described verbally, $S(A, B)$ represents the degree to which $A$ is a subset of $B$.

\section{Pavelka-style fuzzy logic for attribute implications}

\subsection{Formulas and basic semantic notions}

We first recall basic notions related to (fuzzy) attribute implications; details can be found in $[2,4,6]$.

Let $Y$ be a finite set of attributes. A fuzzy attribute implication (over $Y$ ) is an expression $A \Rightarrow B$, where $A, B \in$ $\mathbf{L}^{Y}$ ( $A$ and $B$ are fuzzy sets of attributes). Fuzzy attribute implications (FAIs) are formulas of our logic. Next, we introduce a semantics for FAIs. Due to a lack of space and since it is sufficient for our purposes, we present the semantics in tables with fuzzy attributes only (see Section 1.). Rows and columns of these tables are labeled by objects and their attributes; table entries contain truth degrees from $L$ to which a particular object has a particular attribute. Therefore, a table row labeled by object $x$ represents a fuzzy set $M$ of attributes of object $x: M(y)$ equals the table entry at the crossing of row $x$ and column $y$, i.e. a degree to which $x$ has $y$. The intuitive meaning of $A \Rightarrow B$ being true in a data table with fuzzy attributes is: "if it is true that an object has all attributes from $A$, then it has also all attributes from $B$ '. Formally, for a fuzzy set $M \in \mathbf{L}^{Y}$ of attributes, we define a degree $\|A \Rightarrow B\|_{M} \in L$ to which $A \Rightarrow B$ is true in $M$ by

$$
\|A \Rightarrow B\|_{M}=S(A, M)^{*} \rightarrow S(B, M)
$$

where $S(\cdots)$ denote subsethood degrees, see Section 2 , and $^{*}$ is a hedge which serves as a parameter (see $[2,4,6]$ for details and the role of the hedge). Therefore, $\| A \Rightarrow$ $B \|_{M}$ is a truth degree of "if it is true that the object has all attributes from $A$, then it has all attributes from $B$ ". According to Pavelka-style fuzzy logic, a theory in our logic is a fuzzy set $T$ of formulas, i.e. a fuzzy set of FAIs; a degree $T(A \Rightarrow B)$ is intuitively understood as a degree to which we can assume validity of $A \Rightarrow B$ (e.g. when making deductions from $T$ ). The set $\operatorname{Mod}(T)$ of all models of a theory $T$ is defined by

$$
\begin{gathered}
\operatorname{Mod}(T)=\left\{M \in \mathbf{L}^{Y} \mid T(A \Rightarrow B) \leq\|A \Rightarrow B\|_{M}\right. \\
\text { for each } A \Rightarrow B\} ;
\end{gathered}
$$

each $M \in \operatorname{Mod}(T)$ is called a model of $T$. A degree $\|A \Rightarrow B\|_{T} \in L$ to which $A \Rightarrow B$ semantically follows from a theory $T$ is defined by

$$
\|A \Rightarrow B\|_{T}=\bigwedge_{M \in \operatorname{Mod}(T)}\|A \Rightarrow B\|_{M} .
$$

That is, $\|A \Rightarrow B\|_{T}$ is a degree to which $A \Rightarrow B$ is true in each model of $T$.

Remark 1 Note that for $\mathbf{L}=\mathbf{2}$ (ordinary case in which 0 and 1 are the only truth degrees), the above notions yield their well-known ordinary counterparts, see e.g. [8, 12]. Note also that our extension to a fuzzy setting gives much more expressive power in expressing data dependencies; due to lack of space we refer the reader to the references.

\subsection{Deduction rules and provability}

In Pavelka-style fuzzy logic, one works with truthweighted formulas. Accordingly, we consider weighted FAIs, i.e. pairs $\langle A \Rightarrow B, a\rangle$ where $A \Rightarrow B$ is a FAI and $a \in L$ is a truth degree. Weighted FAIs will be used in making deductions from theories $T$, i.e. from possibly partially true FAIs. For simplicity, we denote (schemes of) FAIs by $\varphi$ and the like in the subsequent text. Furthermore, $T$ denotes a theory (fuzzy set of FAIs).

Our deduction rules work with weighted FAIs; each $n$-ary rule $R$ will be visualized by

$$
\frac{\left\langle\varphi_{1}, a_{1}\right\rangle, \ldots,\left\langle\varphi_{n}, a_{n}\right\rangle}{\langle\varphi, a\rangle}
$$

which reads: from $\left\langle\varphi_{1}, a_{1}\right\rangle, \ldots,\left\langle\varphi_{n}, a_{n}\right\rangle$ infer $\langle\varphi, a\rangle$. An $n$-ary rule $R$ has two parts, a syntactic part $R_{\text {syn }}$ and a semantic part $R_{\text {sem }} . R_{\text {syn }}$ is a partial $n$-ary mapping assigning a FAI $R_{\text {syn }}\left(\varphi_{1}, \ldots, \varphi_{n}\right)$ to each $n$-tuple $\left\langle\varphi_{1}, \ldots, \varphi_{n}\right\rangle$ of FAIs for which $R_{\text {syn }}$ is defined; $R_{\text {sem }}$ is an $n$-ary mapping assigning a truth degree $R_{\text {sem }}\left(\left\langle\varphi_{1}, a_{1}\right\rangle, \ldots,\left\langle\varphi_{n}, a_{n}\right\rangle\right)$ to each $n$-tuple $\left\langle\left\langle\varphi_{1}, a_{1}\right\rangle, \ldots,\left\langle\varphi_{n}, a_{n}\right\rangle\right\rangle$ of weighted FAIs. Note that we have $\varphi=R_{\text {syn }}\left(\varphi_{1}, \ldots, \varphi_{n}\right)$ and $a=$ $R_{\text {sem }}\left(\left\langle\varphi_{1}, a_{1}\right\rangle, \ldots,\left\langle\varphi_{n}, a_{n}\right\rangle\right)$ above. Note also that one usually assumes that $R_{\mathrm{sem}}\left(\left\langle\varphi_{1}, a_{1}\right\rangle, \ldots,\left\langle\varphi_{n}, a_{n}\right\rangle\right)$ depends 
only on $a_{1}, \ldots, a_{n}$ in Pavelka-style fuzzy logic (we therefore allow for a more general case). An example is a rule of modus ponens in Paveka-style [14]: from $\langle\varphi, a\rangle$ and $\langle\varphi \Rightarrow \psi, a\rangle$ infer $\langle\psi, a \otimes b\rangle$. Before showing our deduction rules, we proceed with further notions adopted from a general setting of Pavelka-style fuzzy logic.

Let $\mathcal{R}$ be a given set of deduction rules. A (weighted) proof of $\langle\varphi, a\rangle$ from $T$ is a sequence $\left\langle\varphi_{1}, a_{1}\right\rangle, \ldots,\left\langle\varphi_{n}, a_{n}\right\rangle$ of weighted FAIs such that $\langle\varphi, a\rangle=\left\langle\varphi_{n}, a_{n}\right\rangle$ and for each $i=1, \ldots, n$ we have $a_{i}=T\left(\varphi_{i}\right)$ or $\left\langle\varphi_{i}, a_{i}\right\rangle$ is obtained by some of the rules of $\mathcal{R}$ from some $\left\langle\varphi_{j}, a_{j}\right\rangle$ 's $(j<i)$. A degree $|A \Rightarrow B|_{T}$ of provability of a FAI $A \Rightarrow B$ from $T$ is defined by

$$
|A \Rightarrow B|_{T}=\bigvee\{a \mid \ldots,\langle A \Rightarrow B, a\rangle \text { is a proof from } T\}
$$

A theory $T$ is called syntactically closed w.r.t. $R$ if for each rule $R \in \mathcal{R}$ and all FAIs $\ldots, \varphi_{i}, \ldots$ for which $R_{\text {syn }}$ is defined we have

$$
R_{\text {sem }}\left(\ldots,\left\langle\varphi_{i}, T\left(\varphi_{i}\right)\right\rangle, \ldots\right) \leq T\left(R_{\text {syn }}\left(\ldots, \varphi_{i}, \ldots\right)\right),
$$

i.e., if a FAI can be syntactically inferred from $T$, then it is in $T$ already. A theory $T$ is called semantically closed if for each FAI $\varphi$ we have

$$
\|\varphi\|_{T} \leq T(\varphi)
$$

i.e., if a FAI follows semantically from $T$, then it is in $T$ already. One can denote by $\operatorname{syn}(T)$ the least syntactically closed theory containing $T$ and by $\operatorname{sem}(T)$ the least semantically closed theory containing $T$. A rule $R$ preserves suprema if $\bigvee_{i} R_{\mathrm{sem}}\left(\ldots,\left\langle\varphi, a_{i}\right\rangle, \ldots\right)=$ $R_{\text {sem }}\left(\ldots,\left\langle\varphi, \bigvee_{i} a_{i}\right\rangle, \ldots\right)$. Then we have

Theorem 2 For any set $\mathcal{R}$ of rules which preserve supre$m a,(\operatorname{syn}(T))(A \Rightarrow B)=|A \Rightarrow B|_{T}$; $(\operatorname{sem}(T))(A \Rightarrow B)=\|A \Rightarrow B\|_{T}$.

\subsection{Soundness and completeness}

Our logic uses the following deduction rules:

$$
\begin{array}{cc}
\text { (Ax) } \frac{}{\langle A \cup B \Rightarrow A, 1\rangle}, \quad \text { (Cut) } \frac{\langle A \Rightarrow B, a\rangle,\langle B \cup C \Rightarrow D, b\rangle}{\left\langle A \cup C \Rightarrow D, a^{*} \otimes b\right\rangle}, \\
\text { (Mul) } \frac{\langle A \Rightarrow B, a\rangle}{\left\langle c^{*} \otimes A \Rightarrow c^{*} \otimes B, a\right\rangle}, \quad \text { (Sh) } \frac{\langle A \Rightarrow B, a\rangle}{\langle A \Rightarrow C, S(C, a \otimes B)\rangle},
\end{array}
$$

for each $A, B, C, D \in \mathbf{L}^{Y}$, and $a, b, c \in L ; S(\cdots)$ denotes a subsethood degree, see Section 2. The rules are inspired by Armstrong axioms, see [12]. Note that, in fact, $(\mathrm{Sh})$ is a parameterized rule; we have one rule $\left(\mathrm{Sh}_{C}\right)$ for each $C$.

As usual, we call a rule (3) derivable from a set $\mathcal{R}$ of rules if there is a weighted proof of $\langle\varphi, b\rangle$ with $a \leq b$ from $T=\left\{{ }^{a_{1}} / \varphi_{1}, \ldots,{ }^{a_{n}} / \varphi_{n}\right\}$ using rules from $\mathcal{R}$ (for each $\left.\varphi_{i}, a_{i}\right)$.

Lemma 3 The following are rules are derivable from (Ax)-(Sh):
(Ref) $\overline{\langle A \Rightarrow A, 1\rangle}$, (Wea) $\frac{\langle A \Rightarrow B, a\rangle}{\langle A \cup C \Rightarrow B, a\rangle}, \quad$ (Pro) $\frac{\langle A \Rightarrow B \cup C, a\rangle}{\langle A \Rightarrow B, a\rangle}$,

(Add) $\frac{\langle A \Rightarrow B, a\rangle,\langle A \Rightarrow C, b\rangle}{\langle A \Rightarrow B \cup C, a \wedge b\rangle}, \quad$ (Tra) $\frac{\langle A \Rightarrow B, a\rangle,\langle B \Rightarrow C, b\rangle}{\left\langle A \Rightarrow C, a^{*} \otimes b\right\rangle}$,

$(\mathrm{Sh} \uparrow) \frac{\langle A \Rightarrow B, a\rangle}{\langle A \Rightarrow a \otimes B, 1\rangle}, \quad(\mathrm{Sh} \downarrow) \frac{\langle A \Rightarrow B, 1\rangle}{\langle A \Rightarrow a \rightarrow B, a\rangle}$,

for each $A, B, C \in \mathbf{L}^{Y}$, and $a, b \in L$.

Proof. Sketch (details postponed to a full version): (Ref): Directly by putting $B=\emptyset$ in (Ax). (Wea): Apply (Cut) to $\langle C \cup A \Rightarrow A, 1\rangle$ (infer this by (Ax)) and $\langle A \cup A \Rightarrow B, a\rangle$. (Pro): Apply (Sh) and observe that $a \leq S(B, a \otimes(B \cup C))$. (Tra): Use (Cut). (Sh $\uparrow):$ Put $C=a \otimes B$ and observe that $1=S(a \otimes B, a \otimes B)$. (Sh $\downarrow)$ : Put $C=a \rightarrow B$ and observe that $a \leq S(a \rightarrow B, 1 \otimes B)$. (Add): Use (Sh $\uparrow)$ to infer $\langle A \Rightarrow a \otimes B, 1\rangle$ from $\langle A \Rightarrow B, a\rangle$; use (Wea) to infer $\langle A \cup a \otimes B \Rightarrow C, b\rangle$ from $\langle A \Rightarrow C, b\rangle$ and then use (Sh $\uparrow$ ) to infer $\langle A \cup a \otimes B \Rightarrow b \otimes C, 1\rangle$; then apply (Cut) to the last pair and to $\langle b \otimes C \cup a \otimes B \Rightarrow b \otimes C \cup a \otimes B, 1\rangle$ to infer $\langle a \otimes B \cup A \Rightarrow b \otimes C \cup a \otimes B, 1\rangle$; then use (Cut) to infer $\langle A \Rightarrow b \otimes C \cup a \otimes B, 1\rangle$, use $(\mathrm{Sh} \downarrow)$ to infer $\langle A \Rightarrow$ $(a \wedge b) \rightarrow b \otimes C \cup a \otimes B, a \wedge b\rangle$, and finally use (Pro) to infer $\langle A \Rightarrow B \cup C, a \wedge b\rangle$ (observe that $B \cup C \subseteq(a \wedge b) \rightarrow$ $b \otimes C \cup a \otimes B)$.

A rule $R$ is called sound if for each $\varphi_{1}, \ldots$ (for which $R_{\text {syn }}$ is defined) and for each $a_{1}, \ldots \in L$ we have

$\operatorname{Mod}\left(\left\{a_{1} / \varphi_{1}, \ldots\right\}\right) \subseteq \operatorname{Mod}\left(\left\{R_{\mathrm{sem}}\left(\left\langle\varphi_{1}, a_{1}\right\rangle, \ldots\right) / R_{\text {syn }}\left(\varphi_{1}, \ldots\right)\right\}\right)$,

i.e., for arbitrary $M$ : if each $\varphi_{1}, \ldots$ is true in $M$ at least to degree $a_{1}, \ldots$, then $R_{\text {syn }}\left(\varphi_{1}, \ldots\right)$ is true in $M$ at least to degree $R_{\text {sem }}\left(\left\langle\varphi_{1}, a_{1}\right\rangle, \ldots\right)$.

Lemma 4 Each of the rules $(\mathrm{Ax})-(\mathrm{Sh})$ is sound. Therefore, (Ref)-( $\mathrm{Sh} \downarrow)$ are sound as well.

Proof. Soundness of (Ax)-(Sh) can be verified by definition. Soundness of (Ref)-(Sh $\downarrow)$ is due to Lemma 3 .

Remark 5 Note that replacing $a^{*} \otimes b$ by $a \otimes b$ in (Cut) and (Tra) would not yield sound rules (counterexample omitted due to lack of space).

The following theorems show soundness and completeness of our logic for FAIs. Note that provability refers to rules (Ax)-(Sh), i.e. $|A \Rightarrow B|_{T}$ is a degree of provability using rules $(\mathrm{Ax})-(\mathrm{Sh})$.

Theorem 6 (soundness) For each fuzzy set $T$ of FAIs and each FAI $A \Rightarrow B$ we have $|A \Rightarrow B|_{T} \leq\|A \Rightarrow B\|_{T}$.

Proof. It can be shown that since each of $(\mathrm{Ax})-(\mathrm{Sh})$ is sound (Lemma 4), each semantically closed theory is also syntactically closed. Since $\operatorname{sem}(T)$ is semantically closed, it is also syntactically closed and so $\operatorname{syn}(\operatorname{sem}(T))=$ $\operatorname{sem}(T)$. Therefore, $|A \Rightarrow B|_{T} \leq(\operatorname{syn}(T))(A \Rightarrow B) \subseteq$ $(\operatorname{syn}(\operatorname{sem}(T)))(A \Rightarrow B)=(\operatorname{sem}(T))(A \Rightarrow B)=\|A \Rightarrow B\|_{T}$ 
Theorem 7 (completeness) Let $L$ be finite. For each fuzzy set $T$ of FAIs and each FAI $A \Rightarrow B$ we have $\| A \Rightarrow$ $B \|_{T}=|A \Rightarrow B|_{T}$.

Proof. " $\geq$ " follows from Theorem 6. " $\leq$ ": Analogously to the proof of Theorem 6, it suffices to show that each syntactically closed theory is also semantically closed. We concentrate on this and present a sketch of proof. Let $T$ be syntactically closed. We need to show $\operatorname{sem}(T) \subseteq T$. Assume, by way of contradiction, that $\| A \Rightarrow$ $B \|_{T} \not \leq T(A \Rightarrow B)$. It is now sufficient to show that there exists a model $A^{+}$of $T$ such that $\|A \Rightarrow B\|_{A^{+}} \leq T(A \Rightarrow B)$ since then, $\|A \Rightarrow B\|\left\|_{T} \leq\right\| A \Rightarrow B \|_{A^{+}} \leq T(A \Rightarrow B)$ is a contradiction with our assumption.

To this end, put $A^{+}=\bigcup_{B \in \mathbf{L}^{\mathbf{Y}}} T(A \Rightarrow B) \otimes B$. Observe first that $T\left(A \Rightarrow A^{+}\right)=1$. Indeed, since $T$ is syntactically closed, (Sh $\uparrow)$ applied to $\langle A \Rightarrow B, T(A \Rightarrow B)\rangle$ yields $1=T(A \Rightarrow T(A \Rightarrow B) \otimes B)$. Since both $Y$ and $L$ are finite, repeated application of (Add) and syntactical closedness of $T$ yields $T\left(A \Rightarrow A^{+}\right)=1$.

We now verify (a) $\|A \Rightarrow B\|_{A^{+}} \leq T(A \Rightarrow B)$ and (b) $A^{+}$is a model of $T$.

(a): Observe first that $A \subseteq A^{+}$and thus $S\left(A, A^{+}\right)^{*}=1$. Indeed, syntactical closedness of $T$ applied to (Ref) yields $T(A \Rightarrow A)=1$ and thus $A^{+} \supseteq T(A \Rightarrow A) \otimes A=A$. Now, $\|A \Rightarrow B\|_{A^{+}}=S\left(A, A^{+}\right)^{*} \rightarrow S\left(B, A^{+}\right)=1 \rightarrow S\left(B, A^{+}\right)=$ $S\left(B, A^{+}\right)$. We therefore need to show $S\left(B, A^{+}\right) \leq T(A \Rightarrow$ $B)$. As $T$ is syntactically closed, we get $S\left(B, A^{+}\right) \leq$ $T\left(A^{+} \Rightarrow B\right)$ (hint: use (Ax) to infer $\left\langle A^{+} \Rightarrow S\left(B, A^{+}\right) \otimes\right.$ $B, 1\rangle$, then ( $\operatorname{Sh} \downarrow)$ with $a=S\left(B, A^{+}\right)$, then observe $B \subseteq$ $S\left(B, A^{+}\right) \rightarrow S\left(B, A^{+}\right) \otimes B$ and use (Pro)). Then, (Tra) applied to $A \Rightarrow A^{+}$and $A^{+} \Rightarrow B$ and syntactical closedness of $T$ gives $S\left(B, A^{+}\right) \leq T(A \Rightarrow B)$, proving (a).

(b): We need to show $T(C \Rightarrow D) \leq\|C \Rightarrow D\|_{A^{+}}$for any $C \Rightarrow D$, which is equivalent to $S\left(C, A^{+}\right)^{*} \otimes T(C \Rightarrow$ $D) \otimes D \subseteq A^{+}$. Since $T\left(A \Rightarrow A^{+}\right)=1$, it suffices to show that $T\left(A \Rightarrow S\left(C, A^{+}\right)^{*} \otimes T(C \Rightarrow D) \otimes D\right)=1$ (namely, $A^{+}$is the largest $E$ with $T(A \Rightarrow E)=1$, for if $E \supset A^{+}$ for such an $E$, then $A^{+}=\bigcup_{B} T(A \Rightarrow B) \otimes B \supseteq T(A \Rightarrow$ $E) \otimes E=E$, a contradiction). To see this, it is enough to show $T(C \Rightarrow D) \leq T\left(A \Rightarrow S\left(C, A^{+}\right)^{*} \otimes D\right)$ (use (Sh $\uparrow$ ), we omit details). Since $T$ is syntactically closed, we have $T\left(A \Rightarrow A^{+}\right)=1$ and $T\left(A^{+} \Rightarrow S\left(C, A^{+}\right)^{*} \otimes C\right)=1$ (by (Ax) since $A^{+} \supseteq S\left(C, A^{+}\right)^{*} \otimes C$ ); then using (Tra), $T\left(A \Rightarrow S\left(C, A^{+}\right)^{*} \otimes C\right)=1$; by (Mul) we get $T(C \Rightarrow D) \leq$ $T\left(S\left(C, A^{+}\right)^{*} \otimes C \Rightarrow S\left(C, A^{+}\right)^{*} \otimes D\right)$; applying now (Tra) to the last facts, we get $T(C \Rightarrow D) \leq T\left(A \Rightarrow S\left(C, A^{+}\right)^{*} \otimes\right.$ $D$ ), proving (b).

Remark 8 (1) One can get completeness for arbitrary $\mathbf{L}$ when using a modified version of (Add); details omitted.

(2) Note also that a degree of semantic entailment can be captured by an ordinary notion of a proof (from a set of FAIs) using a technical trick [5]. Compared to this, the present framework naturally fits a fuzzy approach to attribute implications.

(3) The full version of this paper contains full proofs, examples, further rules and discussion about their mutual derivability and other issues.

Acknowledgement Supported by grant No. 1ET101370417 of GA AV ČR, by grant No. 201/05/0079 of the Czech Science Foundation, and by institutional support, research plan MSM 6198959214.

\section{References}

[1] Bělohlávek R.: Fuzzy Relational Systems: Foundations and Principles. Kluwer, Academic/Plenum Publishers, New York, 2002.

[2] Bělohlávek R., Chlupová M., Vychodil V.: Implications from data with fuzzy attributes. In: Proc. AISTA 2004, 5 pages, ISBN 2-9599776-8-8.

[3] Bělohlávek R., Vychodil V.: Fuzzy attribute logic: syntactic entailment and completeness. In: Proc. JCIS 2005, pp. 78-81, ISBN 0-9707890-3-3.

[4] Bělohlávek R., Vychodil V.: Fuzzy attribute logic: attribute implications, their validity, entailment, and non-redundant basis. In: Proc. IFSA 2005, vol. I, pp. 622-627, ISBN 7-302-11377-7.

[5] Bělohlávek R., Vychodil V.: Axiomatizations of fuzzy attribute logic. In: Proc. IICAI 2005, pp. 2178-2193, ISBN 0-9727412-1-6.

[6] Bělohlávek R., Vychodil V.: Attribute implications in a fuzzy setting. In: Missaoui R., Schmid J. (Eds.): ICFCA 2006, LNAI 3874, pp. 45-60, 2006.

[7] Bělohlávek R., Vychodil V.: Data tables with similarity relations: functional dependencies, complete rules and non-redundant bases. In: Lee M. L., Tan K. L., Wuwongse V. (Eds.): DASFAA 2006, LNCS 3882, pp. 644-658, Springer, 2006.

[8] Ganter B., Wille R.: Formal Concept Analysis. Mathematical Foundations. Springer, Berlin, 1999.

[9] Gerla G.: Fuzzy Logic. Mathematical Tools for Approximate Reasoning. Kluwer, Dordrecht, 2001.

[10] Hájek P.: Metamathematics of Fuzzy Logic. Kluwer, Dordrecht, 1998.

[11] Hájek P.: On very true. Fuzzy Sets and Systems 124(2001), 329-333.

[12] Maier D.: The Theory of Relational Databases. Computer Science Press, Rockville, 1983.

[13] Novák V., Perfilieva I., Močkoř J.: Mathematical Principles of Fuzzy Logic. Kluwer, 1999.

[14] Pavelka J.: On fuzzy logic I, II, III. Z. Math. Logik Grundlagen Math. 25(1979), 45-52, 119-134, 447464.

[15] Zhang C., Zhang S.: Association Rule Mining. Models and Algorithms. Springer, Berlin, 2002. 